\title{
A LINK BASED GENETIC ALGORITHM APPROACH IN OPTIMIZING ROUTING IN WIRELESS AD HOC NETWORK
}

\author{
Mohammed Khurram \\ Research Scholar, VTU, Belagavi, Karnataka,India \\ khurramashrafi@gmail.com \\ Dr.Arun Biradar \\ Research Guide,VTU, Belagavi, Karnataka, India \\ hodcsea@gmail.com
}

\begin{abstract}
A Wireless ad-hoc Network is one of the types of decentralized network. Due to its free to roam attribute they may form frequent break in the network topology which slowdowns the performance for the applications. Therefore, it is important to expect the link of the nodes that are under critical state that leads to link break. By introducing Newton's divided difference method, link in the critical state node can be analyze, identified and desire action has been taken. Genetic Algorithm (GA) helps in execution of all these problems. this algorithm forecast the availability time in the link that means a short time before the link breaks; during this valuable time route-repair function repairs the route locally or if not it send the information to source nodes in aid to find a new route well in advance. The performance is carrying out using ns-2.35 network simulator.
\end{abstract}

Keywords: Ad-hoc routing; Newton divided difference; link prediction; Genetic algorithm; network simulation.

\section{Introduction}

Wireless or Mobile Ad-hoc network is a type of infrastructure-less network that forms a temporary network of nodes that are capable of communicating with each other for collected, gathering and information processing. The mobile nodes that forms a network that are not in the range of communication links uses an intermediate node called router to forward packets through multiple hop routing. The nature of the network id Ad-hoc, so the movement of nodes can rapidly changes in different directions that show the way to break the route link, end-toend delay and hence degrade the quality of service of the network. There are different types of existing ad-hoc routing protocol that manages routing, QoS, end to end delay. Proactive and reactive routing protocols are widely used routing protocol [8]. Proactive protocols are table driven routing protocols that provides routing information up-to-date of all the nodes in the beginning itself. The advantage of proactive is that the routes in which path of the destination node will be always know before the route discovery process. In compare to on-demand or reactive routing protocols, the source node will be finding the route on demand. Reactive protocol traffic overhead is less than proactive protocol because it wills also updates the unused paths in the table. The proactive protocol has bears operating cost in maintaining paths and takes lots of delay for creating and re-establishment of paths.

In both the reactive and proactive routing protocols, an alternate path is always required whenever an active path fails. The failure paths detection cost are high in contrast to packet latency, thus whenever the path fails, the packets experience more delays and connectivity of the flow is interrupted lead to establishment of new path. When a source node receive a route breaks it sends out stream node about the link failure or if the node is near to destination node it alters and execute the path and send buffered data through the new node. Hence, in our proposal, A node which are assumes at critical stage this is calculated and identified by Newton's divided difference interpolation algorithm. When a node enters the critical time, the critical message is send to the upstream node that the node is about to break. During this very limited time, Genetic Algorithm (GA) resolves the alternate link or a new path. Genetic Algorithm is used to select best path as a alternate to be discovered or through crossover link node alternate used to continue the buffered packet send to the destination. The techniques of GA's used are recombination, crossover and mutation.

In this paper it has been organized as follows. Section II, explains about overview of reactive protocol, its operation structure. In this paper, AODV protocol is used as reactive protocol. Section III, describe analytical model of identify critical time at which node about to break, here introduce the Newton's divided difference interpolation algorithm in calculating and identifying critical time used in our proposed scheme. Section IV, describes how GA is established and helps during route discovery and Route Maintenance phase. Section V, presents an experimental evaluation of the proposed mechanism and Section VI present concluding remarks. 


\section{Reactive Routing Protocol}

Ad-hoc On demand Distance Vector (AODV) routing protocol is reactive routing protocol which is most studied under research through MANET and this protocol creates the routes on-demand. [4] In AODV, each node maintain routing table, and each routes will ensured with the sequence number and each with routing information. Whenever each node receives a packet on-demand, the routing table information is updated each on the sequence number and on the number of hops. Whenever a route needs to be established from source to destination, it is required to establish two phase such as at the route discovery phase and at the route maintenance phase [15].

\subsection{Route discovery phase}

When a source node wants to start a route to the destination and there is no route information in the routing table, the source node will broadcast to nearby nodes called Route request packet (RREQ) and then intern to intermediate node till it reach the route to the destination node. When the RREQ packet received at the destination node through flooding, it stops further broadcasting and it unicast the route back from destination node to the source node through the packet called Route Reply packet (RREP) which creates and updates in the routing table[4],

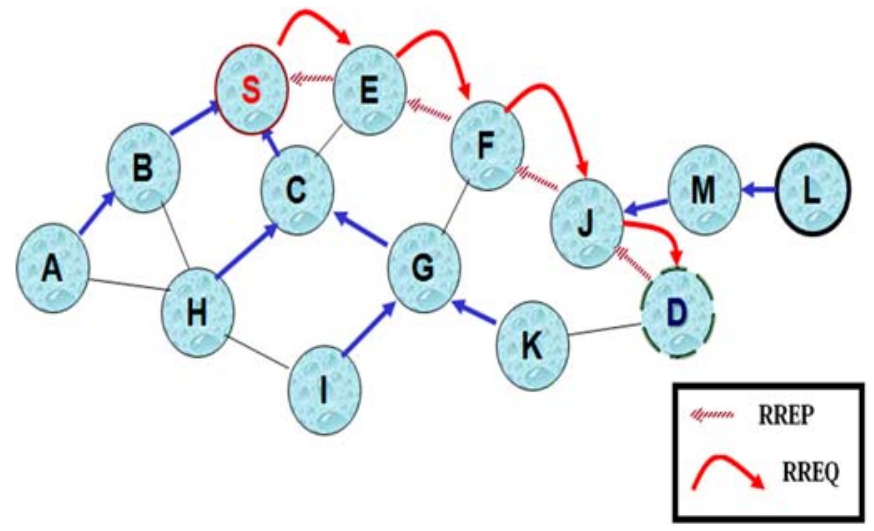

Fig. 1. The process of route discovery.

The RREP packet reverse route has an incremented the sequence number. if the RREQ packet is flooded and cannot find the destination node it rebroadcast the RREQ packet. The process will continued until a source node establish a route or path to the destination node and updates a forward routing table and starts communications. Figure 1 shows the process of the route discovery in which the two steps such as RREQ and RREP packet are considered, here each node that has the valid route through RREQ packet to the destination node in the routing table, hence it unicast the RREP to the source node instead of the destination node. In the AODV route discovery, when the source node $\mathrm{S}$ broadcasts the RREQ to its neighbor node which intern with multi hop reach the destination node $\mathrm{D}$. the destination node $\mathrm{D}$ unicast the RREP through $\mathrm{D} \rightarrow \mathrm{J} \rightarrow \mathrm{F} \rightarrow \mathrm{E} \rightarrow \mathrm{S}$. Whenever each of the node receives already processed RREQ packets, it rejects the RREQ packet in order to avoid looping and hence reduce overhead time.

\subsection{Route maintenance}

In Route Maintenance phase, each node broadcasts a Hello packet in a periodically manner for local network connectivity [14]. Afterwards node broadcasts the RREP messages with TTL $=1$. Whenever the node does not receive any packets from a neighbor node time, it assumes a link break to the neighbor node. Additionally, when the node has confirms link break towards the neighboring node base on an acknowledgment of MAC layer, it notice a route break to the destination node and tells that the neighboring node is next to destination is one hop of the route. If the node that detects the link break is near to a hop or a two to the destination node, it creates alternate path route to the destination node, through Local Repair function. The local repair function in route maintenance is the route discovery at the locally link break. During the local repair, the buffering of arrival data packets is done. When the local repair is successful done and RREP is received at the source, the node restarts the sending data packets in the buffer store. 


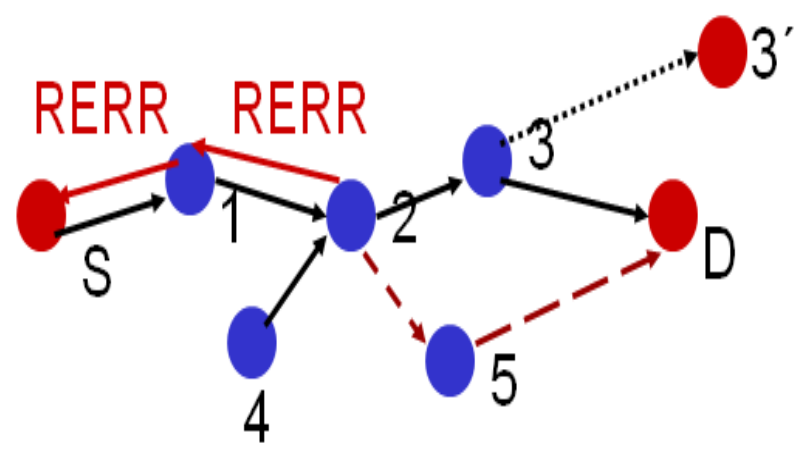

Fig. 2. The processes of route maintenance

In the Figure 2 shows the process of Route Maintenance. In this two processes encountered one is RERR that acknowledge the upstream node that the link has broken inform to the source node to do re-broadcasting for new route. Here in this example node 2 and node 3 link breaks and upstream node 2 inform to node $\mathrm{S}$ to re-broadcast the discovery process. On the other hand, node 2 , which is near to destination, has some time to re-link the path in alternate way and it link with node 5 to destination node $\mathrm{D}$ through local repair function. The buffered arrival data are restored and communication process is back to work.

\section{Analysis of Predictable Time Model}

Routing place an important aspect in MANET routing protocol, since mobility of nodes results in numerous link Routing place an important aspect in MANET routing protocol, since mobility of nodes results in numerous link breaks and thus leads to frequent change in topology of the network. Here, Quality of Service placed is an important issue and becomes a challenge effort. In Routing process, whenever a link break take place, the path of the link has to be repaired internally or it will initiate a new path through route discovery process that lead to packet drop. The network complete process leads to wastage of resource such as Energy, frequent link break with the nodes. For each 'intermediate node that leads to breakup from the upstream has certain factor that inform the link break in danger. The factor involved such as node density, overlapping of the node, energy level and the receiving node[21].

During this process, we are proposing an algorithm that foretell the time, whenever a node active link will break. This is done by calculate approximately the time the data packet at receiving signal strength reduce below threshold power. This means when two nodes are moving apart from each other due to random movement there is a point at which strength of the signal breaks. These predictions of signal strength are crucial part of the route maintenance phase. By considering this crucial time two things can happens, the upstream node repair itself internally through route repair function or creating a new path by notifying the source node. In this process of critical time the link based genetic algorithm is used to optimize the path during route repair and during route discovery. This prediction-based technique can be solved by implementing Newton divided difference method [18]. In this method, the consecutive data packet signal strength is measure from receiving node from nearby link node; here we are measuring last three-packet signal strength using the following formula obtained by Newton interpolation polynomial method.

$$
f(x)=f\left(x_{0}\right)+\left(x-x_{0}\right) f\left(x_{0}, x_{1}\right)+\ldots .+\left(\prod_{i=1}^{n-1}\left(x-x_{i}\right) f\left(x_{0}, x_{1}, \ldots, x_{n}\right)\right.
$$

If the times of incidence of last three packets are $\mathrm{t}_{1}, \mathrm{t}_{2}, \mathrm{t}_{3}$ and the receiving signal strength of the last packet are $S_{1}, S_{2}$ and $S 3$ respectively. Let $S_{r}$ be the threshold value of signal strength obtained and $t_{p}$ be the predicted time obtained when receiving power falls to the threshold power at which the link break process about to be happen. This is the time $t_{p}$ is to be predicting so that the link breaks problem can be rectified and the power with the minimum transmission range received by the node. [1]The standard Wireless LAN have the threshold signal strength $\mathrm{S}_{\mathrm{r}}$ value of $1.26 \times 10^{\wedge} 2 \mathrm{~mW}$ atts approximately and transmission range of maximum $2.4 \mathrm{GHz}$ for the range of $500 \mathrm{mtrs}$. The predictable signal strength of the data packets received can be calculated, where the divided differences of first and second are $\Delta$ and $\Delta^{2}$ respectively [17].

$$
\begin{gathered}
S_{r}=S_{1}+\left(t_{p}-t_{1}\right) \Delta+\left(t_{p}-t_{1}\right)\left(t_{p}-t_{2}\right) \Delta^{2} \\
\mathrm{~S}_{\mathrm{r}}=\mathrm{S}_{1}+\frac{\left(t_{p}-t_{1}\right)\left(S_{2}-S_{1}\right)}{\left(t_{2}-t_{1}\right)}+\left(t_{p}-t_{1}\right)\left(t_{p}-t_{2}\right)\left(\frac{\left(S_{3}-S_{2}\right)}{\left(t_{3}-t_{2}\right)}-\frac{\left(S_{2}-S_{1}\right.}{\left(t_{2}-t_{1}\right)}\right) /\left(t_{3}-t_{1}\right) \\
\text { Let } X=\frac{\left(S_{2}-S_{1}\right)}{\left(t_{2}-t_{1}\right)} \text { and } Y=\left(\frac{\left(S_{3}-S_{2}\right)}{\left(t_{3}-t_{2}\right)}-\frac{\left(S_{2}-S_{1}\right.}{\left(t_{2}-t_{1}\right)}\right) /\left(t_{3}-t_{1}\right)
\end{gathered}
$$


The equation becomes

$$
\mathrm{S}_{\mathrm{r}}=\mathrm{S}_{1}+\left(\boldsymbol{t}_{p}-\boldsymbol{t}_{\mathbf{1}}\right) X+\left(\boldsymbol{t}_{p}-\boldsymbol{t}_{\mathbf{1}}\right)\left(\boldsymbol{t}_{p}-\boldsymbol{t}_{\mathbf{2}}\right) Y
$$

Rearranging equation as

$$
\boldsymbol{Y} \boldsymbol{t}_{p}^{2}+\left(X-Y t_{1}-Y t_{2}\right) t_{p}+\left(S_{1}-\mathrm{S}_{\mathrm{r}}-X t_{1}+t_{1} t_{2} Y\right)=0
$$

This is of the form

$$
\alpha t_{p}^{2}+\beta t_{p}+\gamma=0
$$

Where $\alpha=\mathrm{Y} \beta=\left(X-\boldsymbol{Y} \boldsymbol{t}_{\mathbf{1}}-\boldsymbol{Y} \boldsymbol{t}_{\mathbf{2}}\right)$ and $\boldsymbol{\gamma}=\left(\boldsymbol{S}_{\mathbf{1}}-\mathrm{S}_{\mathrm{r}}-\boldsymbol{X} \boldsymbol{t}_{\mathbf{1}}+\boldsymbol{t}_{\mathbf{1}} \boldsymbol{t}_{\mathbf{2}} \boldsymbol{Y}\right)$

Therefore, the time at which link fail called predicted time tp is

$$
\boldsymbol{t}_{\boldsymbol{p}}=\frac{-\boldsymbol{\beta}+\sqrt{\boldsymbol{\beta}^{2}-4 \alpha \gamma}}{2 \alpha}
$$

If the $t_{p}$ is the predicted time at which is link predicting about to break. The new time called critical time $t_{c}$ is introduced which is smaller than $t_{p}$ which is adequate enough to send warning signal message to upstream node i,e. $t_{c}<t_{p}$. during this critical time $t_{c}$ incorporating link forecasting model in which we use genetic algorithm in order to get optimal path. At the critical time $t_{c}$, the the path that seem to be in critical state and there is an adequate amount of received power to send warning signal message to upstream node to perform an alternate path either by local route repair by setting up an alternate path genetically to reach the destination. If the two nodes moving away from each other and signal is about to drop As two nodes move outwards, signal power of the nodes drops. Source nodes play the route discovery by using genetic cross over and mutation mechanism in order to setup new paths. The area first focus is on link break and local repair when nodes are first crossing over by broken links are need to be repaired locally in fewer hops. The value of $\mathrm{k}=2$, link break can be repaired and set up in two hops similarly $\mathrm{k}=3,4,5$ etc. thus this mechanism of controlling local repair helps in minimizing overhead. The proposed local route repair procedure attempts are done by link based genetic algorithm. The following are the steps needed to be setup in our execution model.

(1) Firstly, by integrating the link based mathematical model Newton interpolation polynomial method that has to be developed in the routing information.

(2) Propose a Genetic Algorithm for quick route discovery and evaluate the performance of the link based Genetic algorithm model. Crossover and mutation design for in order to improve the performance of local repair and rediscovering process.

(3) The existing routing protocol her we have taken AODV protocol has to be done Modification in order to reduce more delay, overhead and maximize the throughput.

\section{Link-based GA Model}

\subsection{Introduction to genetic algorithm}

A genetic algorithm (GA) is evolutionary algorithm obtained from biological means in order to have optimal solutions of the desired problem[16]. The chromosomes consisting of many genotypes create each solution. In every phase of generation, a new set of populations is created by exchanging and combining the chromosomes through the earlier generation. Crossover is also sometimes called mating that referred to as recombination of two offspring. Two chromosomes having larger fitness values are picked from the chromosome collection. The starting point and length of the portion of the chromosomes are being crossed randomly. Hence the two new offspring set are created and there put back into the chromosome pool, optimal one is selected for evaluation through mutation. Mutation is place one of the important steps in genetic operation in which new genetic patterns are initiates into the chromosome pool by modifying some of the genes randomly. This process helps genetic algorithm to search for the new and feasible chromosomes in new areas of chromosome pool. After this process, the multicast tree will be altered and genetic algorithm starts from beginning all the process that include encoding, initial population, evaluation, reproduction, crossover and mutation. The simulation of our study shows that our proposal is an efficient and robust algorithm to optimize the existing routing protocol. 


\subsection{Link-based genetic algorithm}

Link based genetic algorithm start as the critical node enters the critical time [13]. As the node, processing in transferring of packets enters the critical time $t_{s}$; the warning message is send to upstream node about the informing that the nodes is in critical stage and break within a span of time. During this process the genetic algorithm makes effectiveness in route maintenance phase. The phase in the maintenance is divided into 5 phases i,e. encoding, Initial population, selection, reproduction, crossover and mutation as shown in figure below consist of flow of link based genetic algorithm.

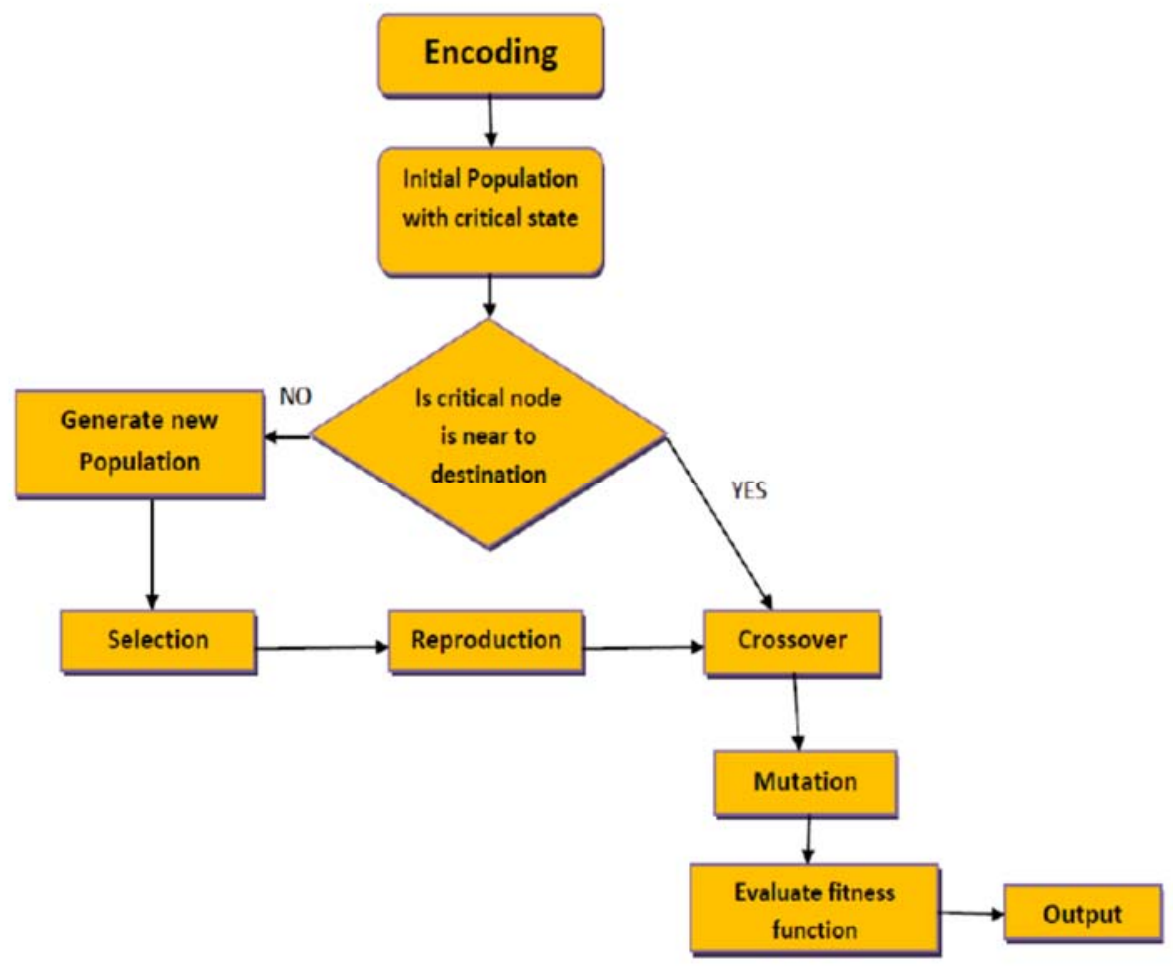

Fig. 3. Process of Link based GA

Initial population and encoding process are one of the starting steps of GA. There are various types of encoding such as edge, vertex and both edge and vertex encoding. Prufer number encoding is used along with the tree, it performs iteratively until two vertices are remained. Initial population is the scheme of creating subset of solutions of during discovery process and defines the set of chromosomes. During this step pseudo code convert multicast tree into two encoding i,e. the sequence and topology encoding. Next the selection process is used to chosen chromosome with fitness values are based on roulette wheel technique and the cost of each node with received power where each times a single chromosome is selected to generate the new offspring[23]. In crossover we make use of two-crossover and it gets selected two chromosomes randomly as parents; the gene chromosome from the two points would be selected and inherited by the offspring. The remaining gene chromosome of the offspring will be inherited from another parent. Mutation is process selecting the gene chromosome by consumption of energy level factor of the nodes[20].

The receiving powers of the nodes are consider in replacing the lower energy node to high energy it can be done using mutation replace function. The optimal energy efficient mutate gene chromosome is identified for the data transfer.

\subsection{Algorithm: Link based genetic algorithm}

Whenever a route discovery process is completed and initial population is encoded; each data packet received at receiving node at each time is recorded and analyze with the following step:

For each neighbor,

Whenever a packet received acknowledge

Update the information (received power, time)

Update the last three packets $p s_{1}, p s_{2}$ and $p s_{3}$ respectively;

If $\left(\left(p s_{1}>p s_{2}\right)\right.$ and $\left.\left(p s_{2}>p s_{3}\right)\right)$ then perform predict() by Newton divided difference method

\{ 
Update the node about critical state obtain by link break

If (existing time $>=t_{c}$ )

\{

Inform message to upstream node about link break is to be happen

Wait for set duration.

Link break warning message is received by upstream node

Perform Routerepair() function with help of genetic algorithm

\}

\}

Routerepair()

\{

Discover path locally to upstream node near to destination node;

If (path is found in fewer hops within set time)

Perform path selection using crossover and mutation.

Else

If (critical time expires)

\{

Inform source node that link break is happen perform route discovery process

Discover path to destination D using new population initialization and encoding;

Find the shortest path with fitness value using selection and reproduction,

Send packet to optimal shortest path with Cost using crossover and mutation.

\}

\}

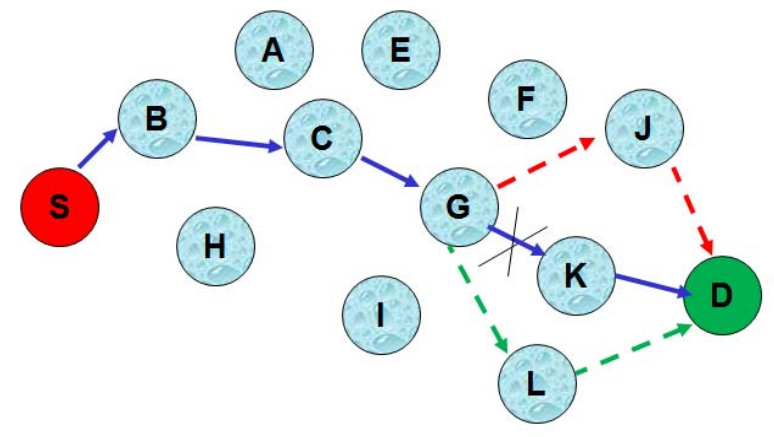

Fig. 4. Route Repair with Link GA

In the figure 4 shows the route repairs with Link GA. A link between Node G and Nod K going to break Node $\mathrm{K}$ is going to entire into critical state at time $t_{c}$. Hereby the node $\mathrm{G}$ is broadcast to find two paths one is from node $\mathrm{J}$ and one is from node L. since using Genetic algorithm node L is the shortest path so the Node $\mathrm{L}$ is connected and form a path establishment. Now the data communication is established the buffer data is exchanged between the source and the destination node

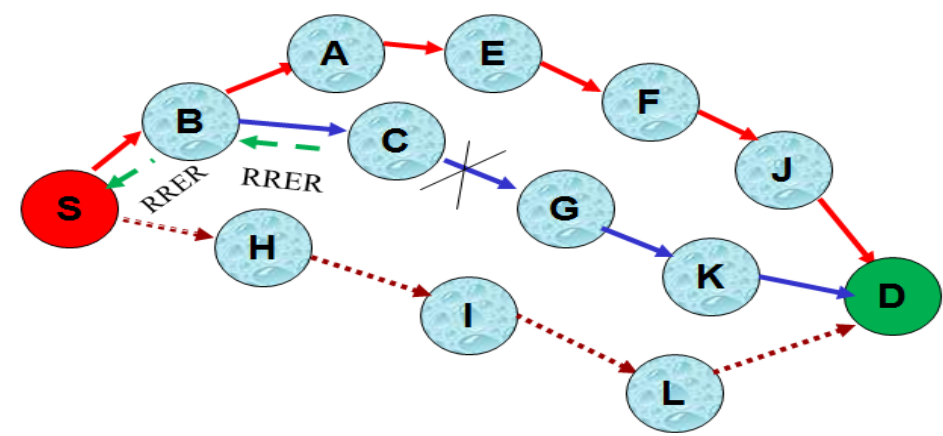

Fig. 5. Route Error with Link GA 
In the figure 5 shows, the critical time $t_{c}$ is expires and route repair operation cannot perform. Now the upstream node $\mathrm{C}$ informs the source node $\mathrm{S}$ to perform route discovery. During this phase, the Genetic algorithm performs the new population and provides the best shortest and cost effective path from source to destination. GA plays an important process such as encoding, selection, crossover and mutation phase that purifies the easiest and most stable path. In the figure there are two path has been established among them the path $S \rightarrow H \rightarrow I \rightarrow L \rightarrow D$ is the most stable path for communication.

\section{Simulation and Results}

In this section, Simulation are carried out with various parameters, each parameter are analyze, evaluated and performance metric computed [2].

\subsection{Simulation parameters}

In this simulation study, we have simulated existing AODV and Link based genetic routing protocol AODVLGA in order to evaluate network performance.[3] Network simulator version Ns2.35 has been used for simulate the results. The mobility model used for network topology is Random waypoint mobility model. Various simulations are carried out and simulation results are obtained. The seed values are added in order to take more number of simulation results; the average of all gives the proper value of evaluation. The detailed simulation studies of parameter shown in table.

Table 1 following are the parameters used for simulation of AODV and AODVLGA

\begin{tabular}{|l|l|}
\hline Simulation traffic & TCP and Constant Bit Rate(CBR) \\
\hline Total number of Connections & $10,20,30,40$ and 50 \\
\hline Simulation Time & 1100 seconds \\
\hline Total number of Nodes & $30,45,60,75,90$ and 120 \\
\hline Maximum velocity & $10,20,25,30,35 \mathrm{mtrs} / \mathrm{sec}$ \\
\hline Pause Time & $10 \mathrm{secs}$ \\
\hline Area of Simulation & $1400 \mathrm{~m}$ by $400 \mathrm{~m}$ \\
\hline Estimated Traffic Load & $5 \mathrm{pkts} / \mathrm{sec}$ \\
\hline
\end{tabular}

\subsection{Performance analysis}

The performance analysis of various parameters in the simulations is evaluate such as average end-to-end delay, packet delivery ratio and route failures $\mathrm{v} / \mathrm{s}$ no. of nodes. The number of nodes is simulated varied from 30 to 120 and velocity of the node varied from 10 to $35 \mathrm{mtrs} / \mathrm{sec}$. In this simulation, CBR simulation traffic model are assumed.

\subsubsection{Average end-to-end delay}

In this simulation, Average end-to-end delay is calculated by taking the difference between data packet time it has generated to the time the data packet is received at its destination.

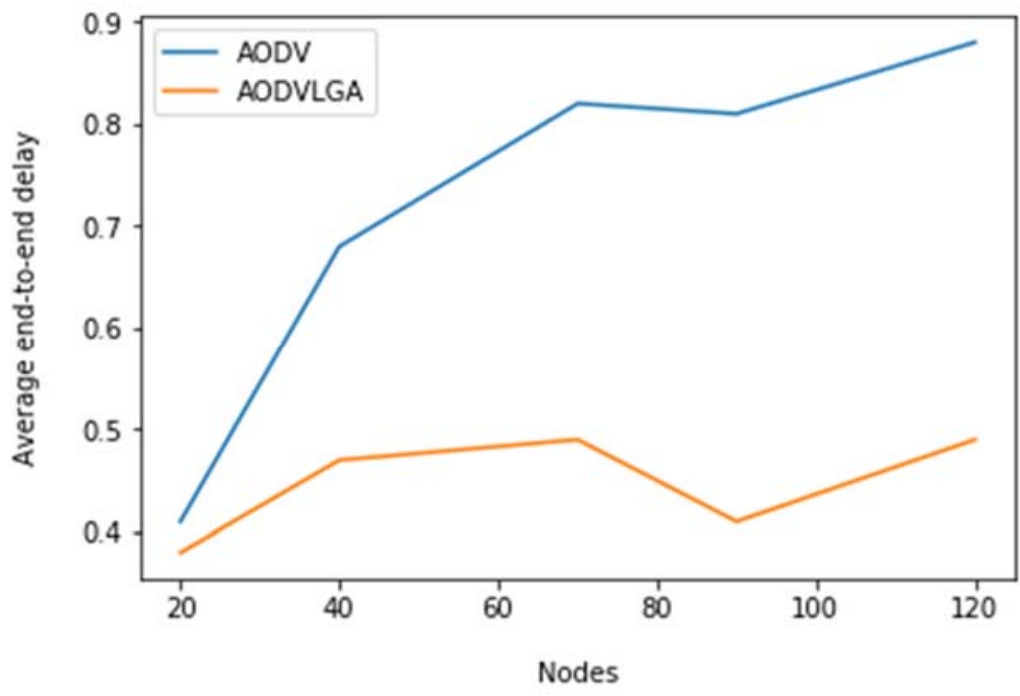

Fig. 6. Showing average end-to-end delay 
Then average of that is average end-to-end delay. In AODVLGA as compared to AODV is seen the decrease in end-to-end delay because of predictive move ahead route discovery in term of route failures. As shown in figure 6 , the decrease in delay is due to retransmission of packets. Conversely, they maybe increase in delay due to increase in network size and increase in collision due to high node density.

\subsubsection{Packet delivery ratio}

Packet delivery ratio is the percentage of data packets successfully received towards the total data packets sent. In this simulation results, the deviation occurs in packet delivery ratio by means of increase in network size. Results show that AODVLGA has better packet delivery ratio compared to AODV. Because AODVLGA has local link repair with GA through which alternate routes identified before the route failures and more buffer data is revert back successfully to the destination.

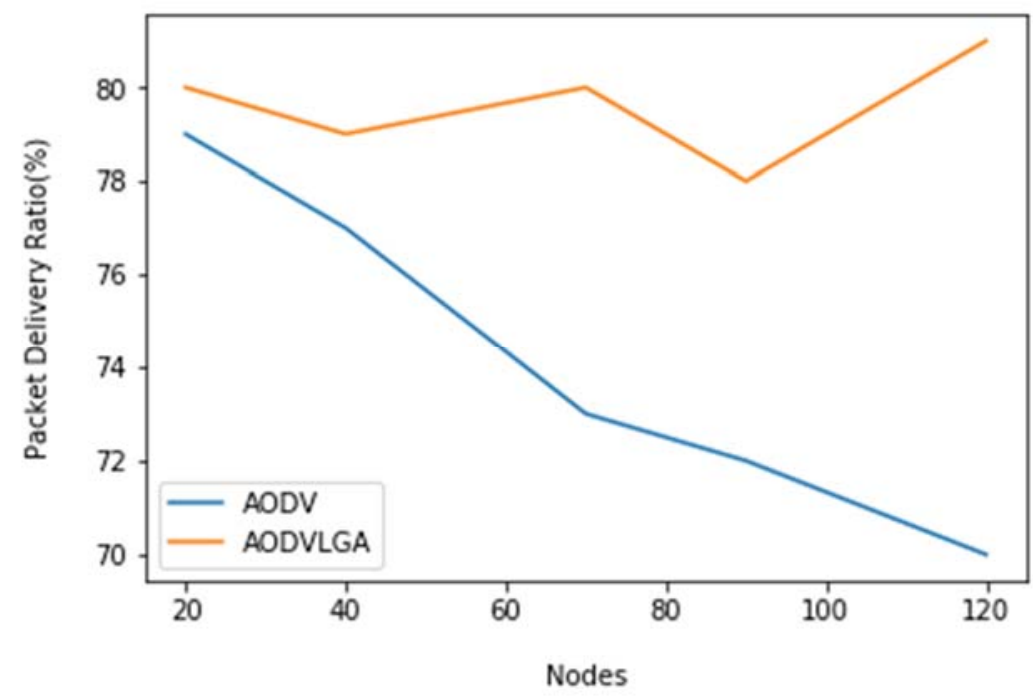

Fig. 7. Showing Packet delivery ratio in percentage

However, In the figure 7, as increase in network size they may be decrease in packet delivery ratio in AODV and AODVLGA because it has frequent route failures which results in packet drops. The increase in node density also has more increase in contentions and collisions. Nevertheless, neighboring nodes or upstream node predicts link break avoids link break and through GA optimal mutate chromosomes increase in packet delivery ratio.

\subsubsection{Routes Failures}

The simulation results are obtained from CBR sources are simulated using routing protocol AODV and AODVLGA.

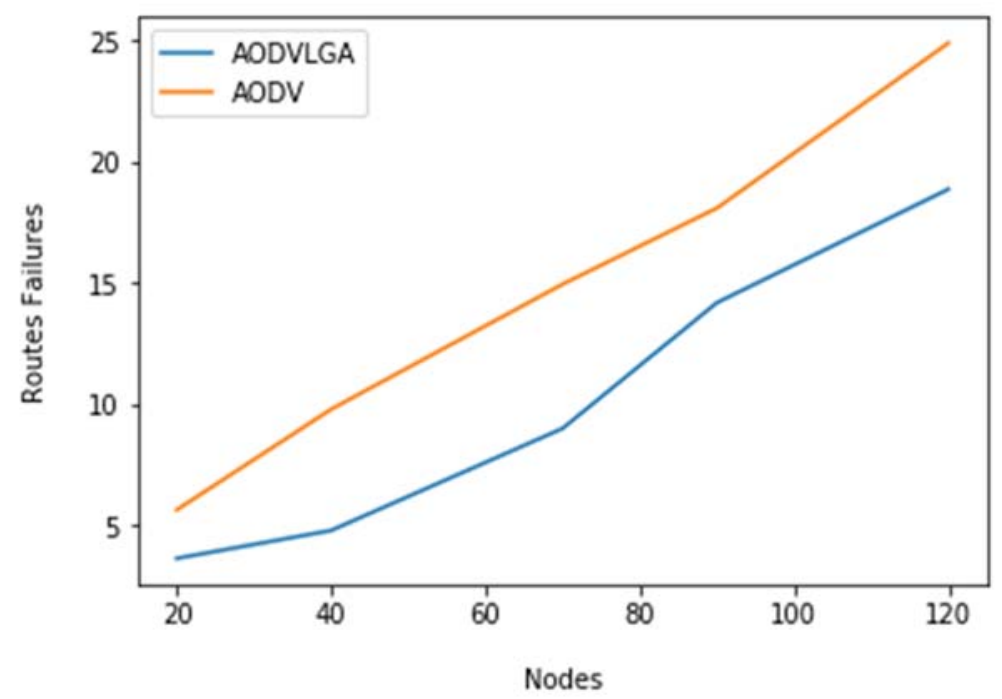

Fig. 8. Route Failures against the nodes 
The graph results shows the variation in route failures is less in AODVLGA while compare to AODV. The network size is kept constant with velocity as 4 meters/second and pause time as 8 seconds. The AODVLGA is less route failure because of two things it avoids route breakage by alternating the route through crossover and mutation and other giving a new optimize route that can retain for longer time. As the availability of additional route in the topology, there are chances of happening frequent switch over. Route failures are also calculate on intentional switchovers.

\section{Conclusion}

In this paper, we have analyzed and improved route maintenance phase. During this phase link based prediction model using Newton difference method is modeled by finding the threshold signal time at which link break about to be happen by calculating last three consecutive received packets signal strength. In this simulation, study AODV routing protocol, which is most frequent routing protocol, used in simulation studies. We have enhanced the AODV with link prediction model with genetic algorithm. AODVLGA is enhanced link based Genetic algorithm compared with AODV using simulations. The AODVLGA protocol improves end-to-end delay and lower routing overhead by limiting the controlled messages and increase higher packet delivery ratio. The AODV and AODVLGA simulated with CBR packets against the higher packet generation rate and with different traffic time. Thus AODVLGA model are use whenever we have frequent link break, rediscovery process and large routing overhead.

\section{References}

[1] WaveLAN/PCMCIA Card User's Guide - Lucent Technologies.

[2] UCB/LBNL/VINT Network Simulator, web-site http://www-mash.CS.Berkeley.EDU/ns.

[3] NS-2 with Wireless and Mobility Extensions, available via web-site http://www.monarch.cs.cmu.edu.

[4] Yih-Chun Hu, Adrian Perrig, and David B. Johnson. "Ariadne: A secure On-Demand Routing Protocol for Ad hoc Networks". MobiCom 2002, September 23-28, 2002, Atlanta, Georgia, USA.

[5] N. Bisnik, "Protocol design for wireless adhoc networks: the cross layer paradigm", Technical Report, ECSE Department, 2005.

[6] V. Srivastava, M. Motani, "Cross layer design: a survey and the road ahead", IEEE Communications Magazine, Vol. 43, no. 12, pp. $112-119,2005$.

[7] M. Conti, G. Maselli, G. Turi, "Cross layering in mobile adhoc network design”, Network Computer, Vol. 37, no. 2, pp. 48-51, 2004.

[8] Siva Ram Murthy and Manoj, Adhoc Wireless Networks Architecture and Protocols, Pearson Education, 2004.

[9] C. R. Lin, "On-Demand QoS Routing in Multi-Hop Mobile Networks", Proceedings of IEEE INFOCOM, Vol. 3, pp. 1735-1744, April 2001.

[10] Y. Chen, Y. Tseng, J. Sheu and P. Kuo, "On-Demand Link-State, Multipath QoS Routing in a Wireless Mobile Adhoc Network", Proceedings of European Wireless, pp. 135-141, February 2002.

[11] G. Ravi, K. R. Kashwan "A new routing protocol for energy efficient mobile applications for ad hoc networks" Computers \& Electrical Engineering, Volume 48, November 2015, Pages 77-85.

[12] P. Mani and D. W. Petr, "Development and performance characterization of enhanced AODV routing for CBR and TCP traffic", Wireless Telecommunications Symposium, 2004.

[13] Prashant Singh, D. K. Lobiyal, "DSR with link prediction using Pareto distribution IEEE International Conference on Networking and Information Technology, pp. 29-33, 2010.

[14] Samir R. Das, Charles E. Perkins, Elizabeth M. Royer. "Performance Comparison of Two On-demand Routing Protocols for Ad Hoc Networks". Proceedings IEEE Infocom page 3-12, March 2000

[15] Yuxia Lin, A. Hamed Mohsenian Rad, Vincent W.S. Wong, and Joo-Han Song. "Experimental Comparisons between SAODV and AODV Routing Protocols". In proceedings of the 1st ACM workshop on Wireless multimedia, 2005.

[16] Yen, Y.S.. "A genetic algorithm for energy-efficient based multicast routing on MANETs", Computer Communications, 2008-0305

[17] Sergio Pastrana, Aikaterini Mitrokotsa, Agustin Orfila, Pedro Peris-Lopez "Evaluation of classification algorithms for intrusion detection in MANETs" Knowledge-Based Systems, Volume 36, December 2012, Pages 217-225

[18] Chib, Reetika, and Amandeep Singh Sandhu. "Enhancing Routing Performance of AODV Protocol using 3rd Ordered Newton's Difference Equation", Indian Journal of Science and Technology, 2016.

[19] M. Malathi, S. Jayashri "Robust against route failure using power proficient reliable routing in MANET" Alexandria Engineering Journal, Volume 57, Issue 1, March 2018, Pages 11-21

[20] Balu Deokate, Chhagan Lal, Denis Trček, Mauro Conti "Mobility-aware cross-layer routing for peer-to-peer networks" Computers \& Electrical Engineering, Volume 73, January 2019, Pages 209-226

[21] Gaurav Singal, Vijay Laxmi, M. S. Gaur, Swati Todi, Riti Kushwaha "Multi-constraints link stable multicast routing protocol in MANETs" Ad Hoc Networks, Volume 63, August 2017, Pages 115-128

[22] A. Chavan, D. S. Kurule, P. U. Dere "Performance Analysis of AODV and DSDV Routing Protocol in MANET and Modifications in AODV against Black Hole Attack" Procedia Computer Science, Volume 79, 2016, Pages 835-844

[23] Rashmi Chaudhry, Shashikala Tapaswi "Optimized power control and efficient energy conservation for topology management of MANET with an adaptive Gabriel graph" Computers \& Electrical Engineering, Volume 72, November 2018, Pages 1021-1036 